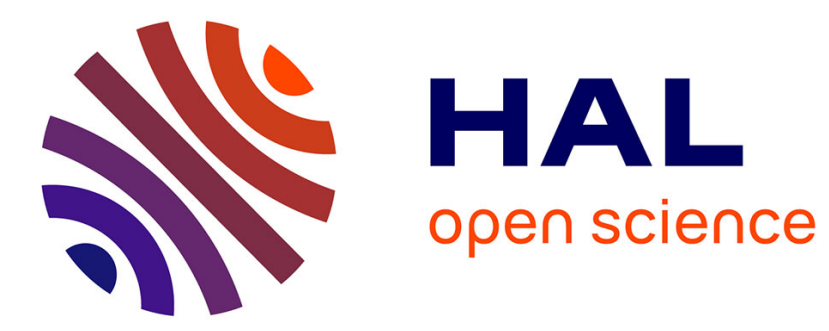

\title{
Theoretical analysis of dynamic response of a vaporizing droplet to acoustic oscillations.
}

\author{
Roger Prud'Homme, Mohammed Habiballah, Lionel Matuszewski, Yves \\ Mauriot, Aurélie Nicole
}

\section{- To cite this version:}

Roger Prud'Homme, Mohammed Habiballah, Lionel Matuszewski, Yves Mauriot, Aurélie Nicole. Theoretical analysis of dynamic response of a vaporizing droplet to acoustic oscillations.. Journal of Propulsion and Power, 2010, 26 (1), pp.74-83. 10.2514/1.39379 . hal-01995198

\section{HAL Id: hal-01995198 \\ https://hal.science/hal-01995198}

Submitted on 19 Apr 2020

HAL is a multi-disciplinary open access archive for the deposit and dissemination of scientific research documents, whether they are published or not. The documents may come from teaching and research institutions in France or abroad, or from public or private research centers.
L'archive ouverte pluridisciplinaire HAL, est destinée au dépôt et à la diffusion de documents scientifiques de niveau recherche, publiés ou non, émanant des établissements d'enseignement et de recherche français ou étrangers, des laboratoires publics ou privés. 


\title{
Theoretical Analysis of Dynamic Response of a Vaporizing Droplet to Acoustic Oscillations
}

\author{
Roger Prud'homme* \\ Pierre et Marie Curie University / Centre National de la Recherche Scientifique \\ 75252 Paris, France \\ Mohammed Habiballah ${ }^{1}$, Lionel Matuszewski ${ }^{2}$, Yves Mauriot ${ }^{3}$ and \\ Aurélie Nicole 4 \\ Office National d'Etudes et de Recherches Aérospatiales, 92322 Châtillon, France
}

\footnotetext{
* Emeritus Research Director, Jean Le Rond d'Alembert Institute, UMR 7190 - Box 162 , 4 place Jussieu - Consultant at Office National d'Etudes et de Recherches Aérospatiales ; roger.prud homme@courriel.upmc.fr

${ }^{1}$ Director of Fundamental and Applied Energetics Department, Office National d'Etudes et de Recherches Aérospatiale (ONERA), 29 avenue de la Division Leclerc, 92322 Châtillon Cedex, France/ Mohammed.Habiballah@onera.fr

${ }^{2}$ Research Scientist ONERA/DEFA - 29 avenue de la Division Leclerc, 92322 Châtillon Cedex, France/ Lionel.Matuszewski@onera.fr

${ }^{3}$ Research Scientist ONERA/DEFA - 29 avenue de la Division Leclerc, 92322 Châtillon Cedex, France/ Yves.Mauriot@onera.fr

${ }^{4}$ Research Scientist ONERA/DEFA - 29 avenue de la Division Leclerc, 92322 Châtillon Cedex, France/ Aurelie.Nicole@onera.fr
}

This paper deals with the analysis of an unsteady evaporating droplet, in connection with combustion instabilities in rocket engines. We first present a bibliographic survey. We then obtain an analytical solution in the frequency domain using the Heidmann analogy of a spherical droplet of constant volume, which represents a mean droplet at a fixed place in a chamber, in the steady regime. For the case of a moderate characteristic time of liquid thermal conduction compared to the droplet lifetime, we show that, for small perturbations, the knowledge of the response factor makes it possible to determine exactly the amplification zone in the frequency domain. We first consider the simplification of Heidmann and Wieber, for which the droplet has a uniform temperature. We then present a new analysis, in which the finite thermal diffusivity of the liquid is taken into account. We find strong differences compared to the results of the preceding model. For the purpose of implementation in a numerical code, we derive a thermal transfer model with $\mathbf{n}$ discrete layers. For the case $n=2$ we verify that this model can be optimized by adjusting the volumetric ratios between layers. Finally, the quasi-steady equations of the gas phase are presented in the appendix.

\section{Nomenclature}

$\begin{array}{ll}A, B & =\text { coefficients in the transfer function } \\ \left\{\begin{array}{l}\bar{a}, b, \\ \bar{b}, c, \bar{c}\end{array}\right. & =\text { coefficients defined in Appendix } \\ B_{T}, B_{M} & =\text { Spalding coefficients for heat and mass transfer } \\ c & =\text { chamber (conditions at infinity) } \\ c_{p}, c_{L} & =\text { heat capacity of the gas at constant pressure, heat } \\ D & \text { capacity of the liquid } \\ \mathrm{F} & =\text { mass diffusion coefficient } \\ f & =\text { droplet constituent } \\ h & =\text { height } \\ i & =\text { time index during navigation } \\ j & =\text { index of chemical species } \\ k & =\text { heat conductivity } \\ l & =\text { latent heat per unit mass } \\ \mathrm{L} & =\text { liquid phase }\end{array}$




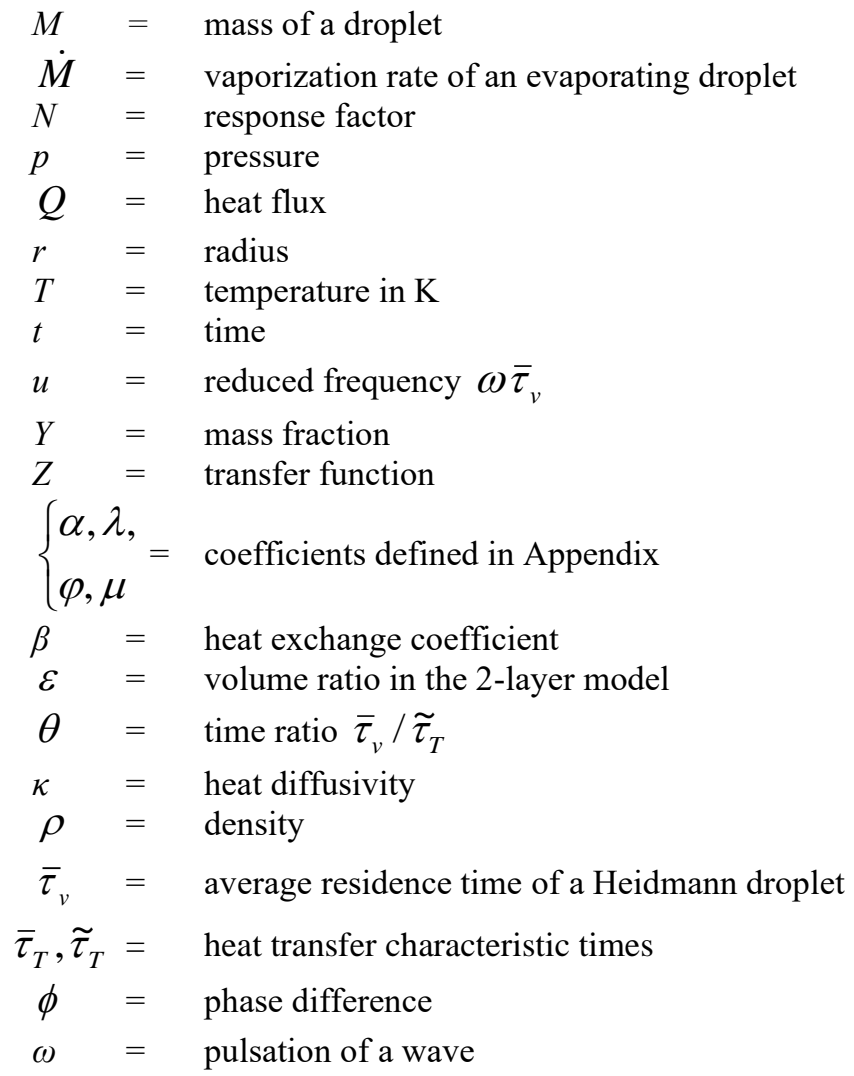

\section{Introduction}

The thermal exchanges inside propellant droplets have an important effect on their transient evaporation behavior and eventually on engine stability. In rocket engines, they occur in particular in the following cases:

1. After injection, fuel or oxidizer liquid droplets are not stabilized. Liquid droplets are generally injected into a gaseous environment having a different temperature, pressure and velocity. A relaxation time is then necessary for a droplet to reach a stabilized situation. At the end of this relaxation period, the velocities of gas and liquid are equal, and the droplets reach a uniform temperature equal to the temperature of saturated vapor at the liquid surface.

Sometimes, the droplet lifetime is too short to reach these stabilized conditions. It is thus possible for them to remain in a transient situation for temperature or/and for velocity.

2. Even for stabilized droplets, acoustic waves generated by the engine may cause departure from the stabilized state. The subsequent vaporization and combustion dynamics can contribute to a high frequency instability of the whole chamber. Indeed, high frequency combustion instability in liquid propellant rocket engines results from a coupling between the combustion processes and the chamber acoustics. It has been shown in previous studies $[1,2]$ that in liquid rocket engines, the acoustic periods of the chamber modes (about $10^{-4}$ to $10^{-3} \mathrm{~s}$ ) may be of the same order of magnitude as the characteristic times of vaporization and combustion, whereas the primary and secondary atomization phenomena intervene at smaller time scales. Many papers about this subject have been published. Let us cite Bhatia \& Sirignano [3], Delplanque \& Sirignano [4,4], DiCicco \& Buckmaster [6], Dubois et al. [7], Duvur et al. [8]; Fachini [9]; Heidmann \& Wieber [10], Heidmann [11], Laroche et al. [12], Sirignano et al. [1]; Tong \& Sirignano [13], Wieber \& Mickelsen [14], Williams [15].

We consider the case of a velocity-stabilized and uniform temperature spherical droplet in an infinite atmosphere. The effect of a non uniform internal temperature has only a minor influence on droplet dynamics (we have verified this fact by an investigation which is not presented in this paper). 
Following the well-known Rayleigh criterion [16], unsteady droplet evaporation and burning could be one possible driving mechanism of instability $[17,18]$. To investigate this possibility, we will look for the response factor, defined as the ratio of evaporating mass flow rate perturbation to the pressure or velocity perturbation.

Of course, the complete prediction of the effect of an acoustic excitation on a burning spray of propellant droplets will need to model properly combustion dynamics. In this study, we will be concerned only with vaporization dynamics. In this study, we will be concerned only with vaporization dynamics. The influence of combustion will be limited to imposing a stationary composition and temperature at infinity. The combined effects of vaporization dynamics and combustion kinetics, and their eventual retroaction on ambient pressure will not be analyzed here.

We will consider an evaporating droplet submitted to an acoustic field with two main objectives:

1) to build a reference analytical linear model for small perturbations (which we will call the "continuous model"), and 2) to build discrete models aimed at treating linear or nonlinear situations in codes for computational fluid dynamics and turbulent combustion.

We will assume that the gas phase is in the quasisteady regime, as was done in many previous investigations of the transient behavior of spherical droplets (see for example Crespo \& Liñan [19], Lefebvre [20], Strahle [21]). This supposes that the gas phase has a very short response time, whereas for the liquid phase, unsteady evolutions must be considered.

\section{Hypotheses of Heidmann and Wieber}

\section{A. Droplet Continuously Fed by a Steady Flow}

In a liquid propellant rocket combustion chamber, the fuel and the oxidizer are usually injected in the form of droplets with a convenient mass flow rate. These droplets then vaporize and the gaseous fuel burns in contact with the gaseous oxidizer. The study of the evolution of moving droplets submitted to an acoustic field during their residence time in the chamber is a complex problem. We therefore adopt a simplified approach, to establish an analytical model in the frequency domain for an isolated droplet. This simplified model can help the comprehension of physical phenomena, and will also be the basis for validating discrete computation fluid dynamics (CFD) models. Following Heidmann and Wieber [10] and Heidmann [11], we adopt the analogy of a constant volume evaporating droplet (at rest with respect to the mean flow, in a first approach), continuously fed by a steady flow. This droplet represents a mean droplet at a specified location in the combustion chamber. In the steady chamber regime, there is a continuous flow of fuel droplets through the combustion chamber. At a given location the mean droplet diameter can be considered as being independent of time, because the droplets are continuously replaced.

The considered evaporating droplet has a constant average radius $\bar{r}_{s}$, and is continuously supplied by a stationary mass flow-rate $\overline{\dot{M}}$ (Fig.1). The droplet can be fed at different places by fuel at the average temperature $\bar{T}_{S}$, with the average mass flow rate $\overline{\dot{M}}$. The total mass balance of the droplet is:

$$
d M / d t=\overline{\dot{M}}-\dot{M}
$$

In steady state, one has: $\dot{M} \equiv \overline{\dot{M}}, d M / d t=0, M=\bar{M}$. If the thermal conductivity of the droplet is infinite, the droplet has a uniform temperature equal to its surface temperature $T_{S}$, independent of the manner in which the droplet is fed.

For case of a finite conductivity the situation is different. In this case two characteristic times intervene: a residence time in the droplet $\bar{\tau}_{v}=\bar{M} / \overline{\dot{M}}$ (which replaces notion of droplet life time in the present situation of constant diameter) and a transfer time for thermal diffusion $\bar{\tau}_{T}={\overline{r_{S}}}^{2} / \kappa_{L}$, where the thermal diffusivity of the liquid is $\kappa_{L}=k_{L} / \rho_{L} c_{L}$. One can estimate that the conduction mode will dominate if $\overline{\tau_{T}}<<\overline{\tau_{v}}$. The case of infinite thermal conductivity of the drop is the limiting case for this 
configuration, and leads to $T_{l} \equiv T_{S}$. On the contrary, for $\overline{\tau_{T}} \gg>\overline{\tau_{v}}$ the convection of fuel will be dominant. The two modes will coexist for $\overline{\tau_{T}} \approx \overline{\tau_{v}}$. We will define the timescale ratio $\theta=9 \bar{\tau}_{v} / \bar{\tau}_{T}$ (the coefficient 9 permits to obtain later a simple expression of the transfer function).
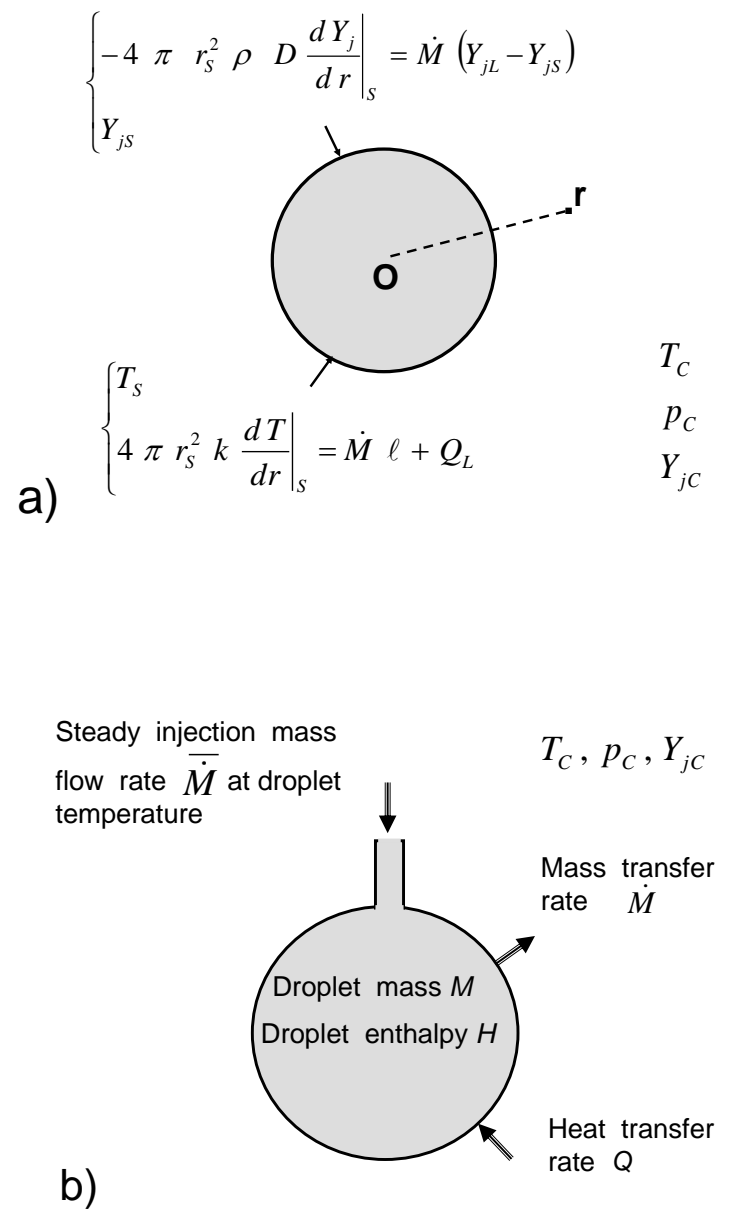

Fig. 1 a) Boundary conditions of a vaporizing droplet. The subscript $C$ designates the conditions at infinity (i.e. the combustion chamber.) b) Droplet of radius $r s$, continuously supplied by a mass flow rate of liquid fuel $\overline{\dot{M}}$.

\section{B. Response Factor}

Heidmann and Wieber [10] considered a droplet submitted to a pressure perturbation. The response is then the resulting heat or mass perturbation. The reduced pressure perturbation is defined as $p^{\prime}=(p-\bar{p}) / \bar{p}$, and the resulting reduced heat or mass perturbation is $q^{\prime}=(q-\bar{q}) / \bar{q}$.

The response factor $\mathrm{N}$ is defined as:

$$
N=\iint_{V, t} q^{\prime}(V, t) p^{\prime}(V, t) d t d V / \iint_{V, t}\left(p^{\prime}(V, t)\right)^{2} d t d \mathrm{~V}
$$

For sinusoidal oscillations, $N=(|\hat{q}| /|\hat{p}|) \cos \phi$, where $|\hat{q}|,|\hat{p}|$ are the moduli and $\phi$ is the phase difference between q' and p'. An example of a droplet submitted to periodic oscillations is the following: the droplet is located at a velocity node and pressure and temperature anti-node of a standing wave in a closed cavity. In this case there is no external flow near the droplet. But the case of a velocity anti-node corresponds to a possible situation and may also be considered. 


\section{The droplet with Uniform Temperature}

The most extreme simplification consists in assuming an infinite thermal conductivity (i.e. $\bar{\tau}_{T}=0$ ), which leads to a droplet temperature that is uniform, but variable in time. We then have (cf. Heidmann and Wieber [10], Chin \& Lefebvre [22]),

$$
M c_{L} \frac{d T_{S}}{d t}=Q_{L}
$$

where $c_{L}$ is the specific heat of the liquid.

Consider now small acoustic perturbations, writing $f=\bar{f}+\Delta f$ where $f$ is a flow parameter, $\Delta f$ is the absolute perturbation, and $f^{\prime}=\Delta f / f$ is the corresponding relative perturbation.

We suppose that the evaporation at the surface is always at equilibrium. However, because the temperature is variable, the partial pressure of species $F$ varies with time, and thus the concentration $Y_{F S}$ adapts itself to the temperature variations. The linearized equation for the heat absorbed by the liquid is simply

$$
\bar{M} c_{L} \bar{T}_{S} d T^{\prime}{ }_{S} / d t=\Delta Q_{L}
$$

For harmonic perturbations of the form $f^{\prime}=\hat{f}(r) e^{i \omega t}$, the last equation becomes

$$
\Delta \hat{Q}_{L}=\bar{M} c_{L} \bar{T}_{S} i \omega \hat{T}_{S}
$$

Eliminating $\Delta \hat{Q}_{L}$ and $\hat{T}_{S}$ between the last equation and the equations for the gas [Eqs. (A.7) and (A.8)], given in the appendix, one obtains the following transfer function:

$$
Z_{1}=\frac{1}{\alpha} \frac{\hat{\dot{M}}}{\hat{p}_{C}}=\frac{i u}{1+i u} \frac{A-i u}{B+i u}
$$

with $u=3 \omega \bar{\tau}_{v}, A=3(\bar{a} \bar{b}-\mu) / \lambda, \quad B=3 \mu / \lambda, \lambda=c_{L} \bar{T}_{S} / \ell$ (the parameters $\bar{a}, \bar{b}, \boldsymbol{\mu}$ and $\alpha$ are defined in the appendix). The real part of $Z_{1}$ and its phase $\phi_{1}$ may be written in an explicit form: $N_{1} / \alpha=u^{2}\left(A B+A+B-u^{2}\right) /\left(1+u^{2}\right)\left(B^{2}+u^{2}\right), \quad \phi_{1}=\pi / 2+\arctan (-u / A)-\arctan (u)-\arctan (u / B)$. Fig. 2 shows the transfer function given by equation (6). The cut-off frequency (corresponding to $N_{1}=0$ ) is given by: $u_{c 1}^{2}=A B+A+B$.

Remark 1: If $\mathrm{A}>0$ and $\mathrm{B}>0$, which is true for all practical cases we have encountered until now, we can deduce that:

1) For the case $\lambda=\frac{c_{L} \bar{T}_{S}}{\bar{\ell}}>>1$, corresponding to a large value of the liquid heat capacity compared to the latent heat, $u_{c 1}^{2} \cong A+B=\frac{3 \bar{a} \bar{b}}{\lambda}$ will have a small positive value, and thus usual frequencies will have the same effect as very high frequencies, so we will have $Z_{1} \cong-1$, meaning that evaporation has a damping effect;

2) In the opposite case $\lambda<<1, u_{c 1}^{2} \cong A B$ will be proportional to $\lambda^{-2}$, and thus have a large positive value; as a consequence for usual frequencies, we will have $\operatorname{Re}\left(Z_{1}\right)>0$, meaning that the evaporation mechanism will have an amplifying effect. 
As a synthesis, we remark that in the case of an infinite internal conductivity, the only coupling mechanism related to the droplet heating is controlled by the ratio $\lambda=\frac{c_{L} \bar{T}_{S}}{\bar{\ell}}$ of the heat capacity to the latent heat. For example, a small value of the heat capacity in comparison to the latent heat will tend to enlarge the amplification range in the frequency domain.

Remark 2: In their analysis, Heidmann \& Wieber [10] suppose that the variations of the latent heat of evaporation $\ell$ with perturbations to the drop temperature are negligible (corresponding to the equation $l^{\prime}=-\frac{2 c}{\overline{T_{S}}-c} T_{S}{ }^{\prime}$, given in the appendix, with $\mathrm{c}=0$ ). They adopt a Sherwood number $S h$ proportional to $\left(r_{s} p_{C}\right)^{1 / 2}$, because they consider the presence of an external flow (in the present case we have $\left.S h=2\right)$. Their expression for the mass flow rate is obtained for equal molar masses $M_{A}=M_{F}$ and for a zero concentration of fuel at infinity, $Y_{F C}=X_{F C}=0$. The Lewis number is taken equal to unity. With these hypotheses, if we substitute the following relations into our equations presented above and in the Appendix: $\quad 1+B_{M}=p_{L} /\left(p_{C}-p_{L}\right), \quad \alpha=\left[p_{L} /\left(p_{C}-p_{L}\right)\right] / \ln \left[p_{C} /\left(p_{C}-p_{L}\right)\right], \quad$ and $\varphi=1+\bar{B}_{M}=\bar{p}_{L} /\left(\bar{p}_{C}-\bar{p}_{L}\right)$ (Cf. the Appendix for the definitions of $B_{M}, \alpha$ and $\varphi$ ), we recover the results of Heidmann and Wieber [10].

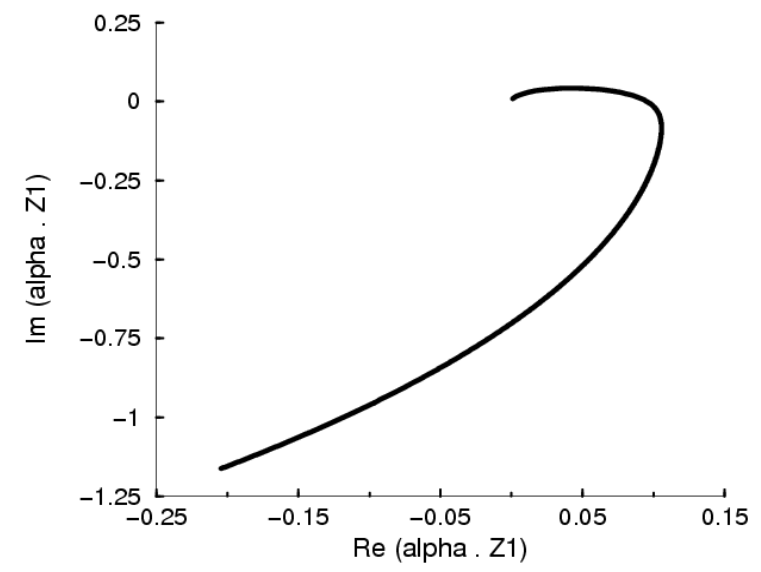

a)

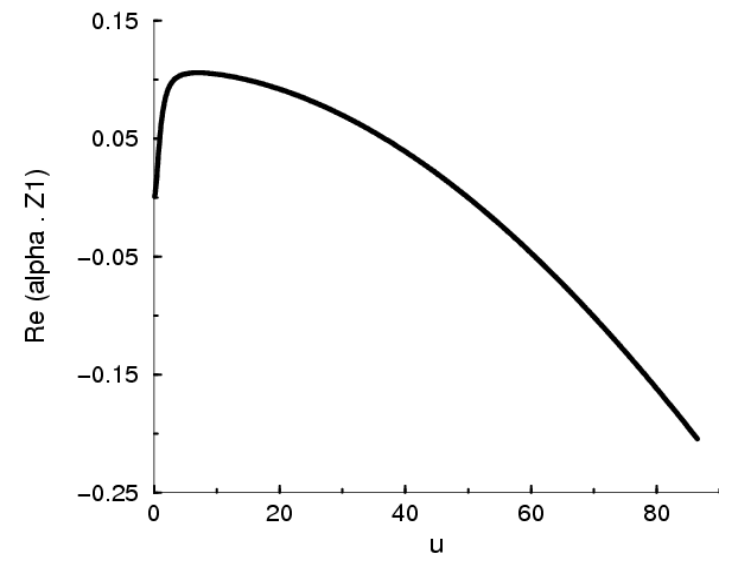

b)

Fig. 2 Oxygen in water vapor at 10 bar and $3200 \mathrm{~K}, Y_{A C}=0.9, A=6.786, B=319.81, \alpha=4.5067$ :

a) $Z_{1}$ function (Eq. 6). b) Real part of $Z_{1}$ as a function of reduced frequency $u$. 


\section{Continuous model with a finite thermal diffusivity}

Let us recall that our first objective is to build an analytical linear reference model for small perturbations. To assess the influence of the thermal wave inside the droplet on the unsteady evaporation mechanism, we abandon the Heidmann \& Wieber [10] hypothesis of infinite liquid conductivity. Studying the thermal exchanges inside the droplet and considering a feeding at the center of the droplet, in the general case the effect of internal convection should be taken into account. However, in order to establish an analytical solution in the frequency domain, we will suppose that the thermal diffusion time is sufficiently small compared to the residence time (or equivalently that the parameter $\theta$ defined in $\S$ II.A must be sufficiently high), that we can neglect the convective term in the energy equation of the droplet.

\section{A. Energy Conservation Equation}

The temperature of the liquid is a function of space and time and verifies the equations

$$
\begin{gathered}
\rho_{L} c_{L} \frac{\partial T_{l}}{\partial t}+\rho_{L} c_{L} v_{r} \frac{\partial T_{l}}{\partial r}-\frac{k_{L}}{r} \frac{\partial^{2}\left(r T_{l}\right)}{\partial r^{2}}=0 \\
\left.4 \pi r_{S}^{2} k_{L} \frac{\partial T_{l}}{\partial r}\right|_{r_{S}}=Q_{L}, \quad T_{l}\left(r_{S}, t\right)=T_{S},\left.\quad \frac{\partial T_{l}}{\partial r}\right|_{r=0}=0
\end{gathered}
$$

The central injection velocity is $v_{r}=\rho_{L} c_{L} \frac{\overline{\dot{M}}}{4 \pi \rho_{L} r^{2}}$, which leads to:

$\frac{\partial\left(r T_{l}\right)}{\partial t}+k_{L}\left(\frac{3}{\theta} \frac{\bar{r}_{s}}{r} \frac{\partial T_{l}}{\partial r}-\frac{\partial^{2}\left(r T_{l}\right)}{\partial r^{2}}\right)=0 \quad$, where we will neglect the convective term, because we have assumed that $\theta=9 \kappa_{L} \bar{\tau}_{v} / \bar{r}_{S}^{2}=9 \bar{\tau}_{v} / \bar{\tau}_{T}>1$.

We thus have: $\rho_{L} c_{L} \frac{\partial T_{l}}{\partial t}-\frac{k_{L}}{r} \frac{\partial^{2}(r T)}{\partial r^{2}}=0,\left.\quad 4 \pi r_{S}^{2} k_{L} \frac{\partial T_{l}}{\partial r}\right|_{r_{S}}=Q_{L}, \quad T_{l}\left(r_{S}, t\right)=T_{S},\left.\quad \frac{\partial T_{l}}{\partial r}\right|_{r=0}=0$

The temperature profiles in a spherical drop were previously obtained numerically or by series expansion, see Law \& Sirignano [23]. Here we need a simple analytical model, and we will use the linearized continuity equations for small perturbations.

\section{B. Linearized Equations of the Continuous Model}

For small perturbations, the equations of energy conservation can be written

$$
\rho_{L} c_{L} \frac{\partial T_{l}^{\prime}}{\partial t}-\frac{k_{L}}{r} \frac{\partial^{2}\left(r T_{l}{ }^{\prime}\right)}{\partial r^{2}}=0,\left.4 \pi r_{S}^{2} k_{L} \bar{T}_{S} \frac{\partial T_{l}{ }^{\prime}}{\partial r}\right|_{r_{S}}=\Delta Q_{L}, T_{l}{ }^{\prime}\left(r_{S}, t\right)=T_{S}{ }^{\prime},\left.\frac{\partial T_{l}{ }^{\prime}}{\partial r}\right|_{r=0}=0
$$

Introducing: $f^{\prime}=\hat{f}(r) e^{i \omega t}$, we find a solution of the form $\quad r \hat{T}_{l}=C\left(e^{s_{0} r}-e^{-s_{0} r}\right)$, with $s_{0}=(1+i) \sqrt{\omega / 2 \kappa_{L}}$ and $C=r_{S} \hat{T}_{S} /\left(e^{s_{0} r_{S}}-e^{-s_{0} r_{S}}\right)$. Thus, we have

$$
\Delta \hat{Q}_{L}=-4 \pi \bar{r}_{S} k_{L} \bar{T}_{S} E \hat{T}_{S}
$$

where $E=1-s_{0} r_{S} \operatorname{coth}\left(s_{0} r_{S}\right)$, with $s_{0} r_{S}=(1+i) \sqrt{3 u / 2 \theta}, \quad \theta=9 \kappa_{L} \bar{\tau}_{v} / \bar{r}_{S}^{2}=9 \bar{\tau}_{v} / \bar{\tau}_{T}$. 


\section{Transfer Function of the Continuous model}

Eliminating $\hat{T}_{S}$ between Eqs. (9), (A.7) and (A.8), we obtain an expression for the complex transfer function $Z=\hat{\dot{M}} / \alpha \hat{p}_{C}$

$$
Z(\theta, u)=\frac{i u}{1+i u} \frac{A+\theta E(u)}{B-\theta E(u)}
$$

where $u, A$ and $B$ have the same definition as in Eq. (6). In the limit of infinitely high values of $\theta$, one has $E \cong-\left(s_{0} r_{S}\right)^{2} / 3=-i u / \theta$ and so $Z \cong i u(A-i u) /(1+i u)(B+i u)=Z_{1}$, the transfer function already found in Sec. II.C for the case of a liquid with infinite thermal conductivity.

In the low frequency limit $u \rightarrow 0, Z \rightarrow 0$ and $N / \alpha=\operatorname{Re}(Z) \rightarrow 0$, and in the high frequency limit $u \rightarrow \infty, Z \rightarrow(-1)$ and $N / \alpha=\operatorname{Re}(Z) \rightarrow(-1)$.

The effect of arbitrarily changing the reduced exchange coefficient $\theta$, is shown in Fig. 3.

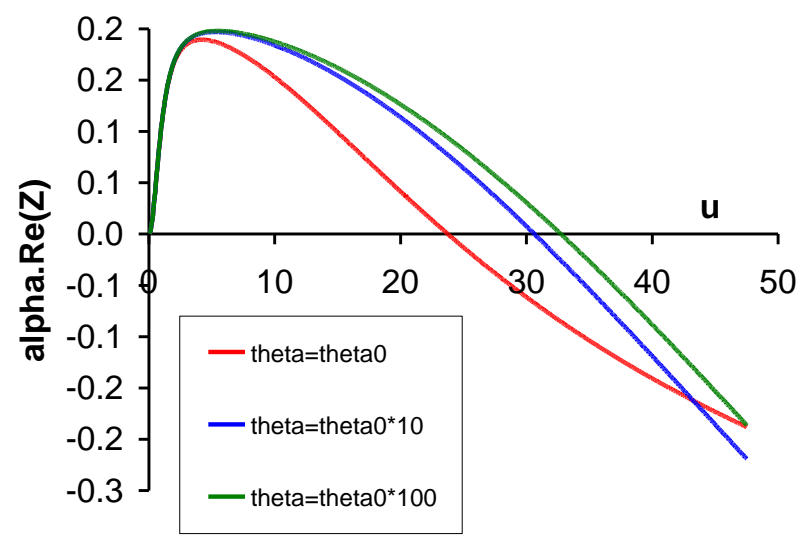

Fig. 3 Influence of the reduced exchange coefficient $\theta$ on the reduced response factor $N / \alpha=\operatorname{Re}(Z)$ of the continuous model in the case of a $\mathrm{LOX}$ droplet in a $\mathrm{O}_{2} / \mathrm{H}_{2} \mathrm{O}$ mixture, $T_{C}=550 \mathrm{~K}, p_{C}=10 \mathrm{~b}, Y_{A C}=0.9, A=10.80$, $B=96.09, \theta_{0}=10.23, \alpha=1.790$

The curves of Fig. 3 show that for each value of the thermal exchange coefficient, the response factor starts from zero at zero frequency, presents a maximum and goes through zero at a cut-off frequency $u_{c}$. Between $u=0$ and $u=u_{c}$, the response factor is positive and the evaporation mechanism has a destabilizing influence. For $u>u_{c}$ the response factor is negative and evaporation has a stabilizing influence. The cut-off frequency $u_{\mathrm{c}}$ is a function of $\theta$. The value of $\theta$ has a significant influence on the extension of the amplification domain. In the case of a liquid oxygen (LOX) droplet in a mixture of gaseous oxygen and water vapor at $\mathrm{T}_{\mathrm{C}}=550 \mathrm{~K}, p_{C}=10 \mathrm{~b}, Y_{A C}=0.9$ (Cf. Fig.3), an increase of $\theta$ (proportional to the thermal conductivity of the liquid) tends to increase $u_{\mathrm{c}}$.

Remark 1: for $\theta \rightarrow \infty$, one finds again for $u_{\mathrm{c}}$ the result obtained in section II.C for a droplet with uniform temperature (Fig. 2b).

Remark 2: the conditions of Fig. 3 have been chosen different from the conditions of Fig. 2, thus the values of the cut-off frequency can not be compared. The reason of this choice was the need to make the 
reduced exchange coefficient $\theta$ sufficiently high, so as to ensure that the hypotheses of the continuous linearized model are valid.

Remark 3: whereas in the case of infinite conductivity the extension of the amplification range is mainly determined by the parameter $\lambda$, in the general case $\theta$ intervenes as a complementary parameter.

Remark 4: the above-mentioned results are in accordance with the feeling that increasing the dissipative phenomena inside the droplet will have a stabilizing effect, in comparison to the nondissipative case $(\theta \rightarrow \infty)$. Moreover, they confirm the need to model adequately the nonstationary heat transfer inside the droplet.

\section{Multi layer model}

Our second objective is to build a discrete multi-layer model (called n-layer model) which can be implemented in a CFD code. Indeed, the continuous analytical model, presented above, can only be used in the limit of small perturbations, for $\theta \gg 1$, and in the framework of frequency domain calculations. We will now look for a linearized form of the n-layer model to validate it by comparison with the continuous model. However, we intend to use it finally in a non linearized form in a CFD code, in order to be able to treat strong perturbations leading to nonlinear responses. Moreover, let us clarify that we intend to implement the n-layer model into a CFD code without the hypothesis of constant diameter.

\section{A. $n$-layer model}

The assumptions of the $n$-layer model are the following: 1) $n$ layers of homogeneous temperature, and 2) Uniform internal conductivity inside the droplet.

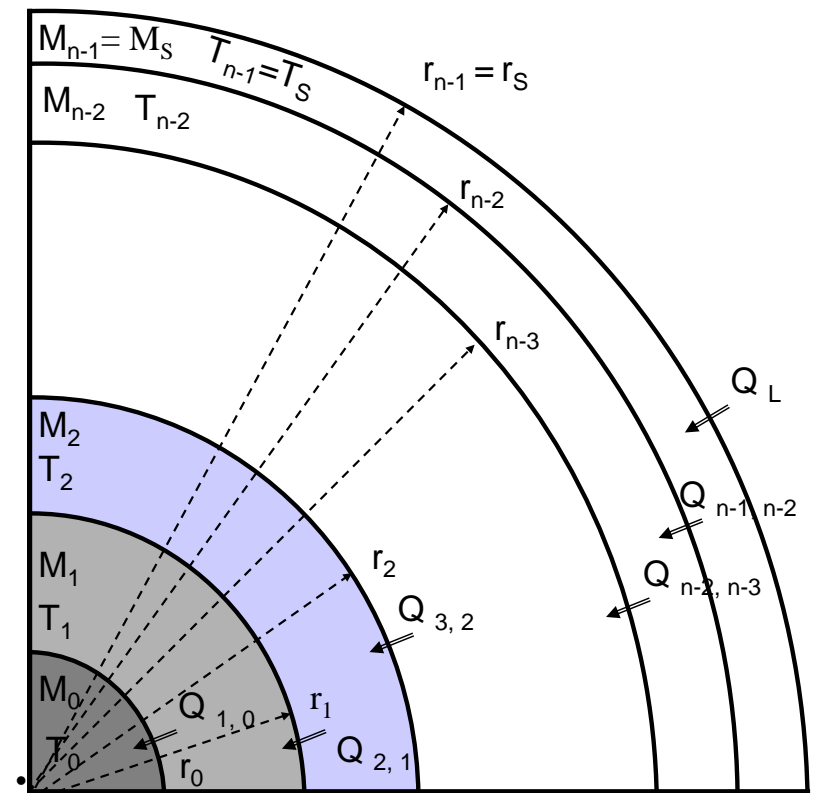

Fig. 4 Heat exchanges in the n-layer model.

\section{Balance equations}

The equations of the model are (Fig.4)

$$
\begin{aligned}
& M_{0} c_{L} \frac{d T_{0}}{d t}=Q_{1,0}, M_{1} c_{L} \frac{d T_{1}}{d t}=Q_{2,1}-Q_{1,0}, \cdots, M_{n-2} c_{L} \frac{d T_{n-2}}{d t}=Q_{n-1, n-2}-Q_{n-2, n-3} \\
& M_{S} c_{L} \frac{d T_{S}}{d t}=-Q_{n-1, n-2}+Q_{L}, M_{S}=M_{n-1}, T_{S}=T_{n-1}
\end{aligned}
$$

The fluxes $Q_{j, j-1}$ can be written: 


$$
\begin{aligned}
& Q_{1,0}=\beta_{0} M_{0} c_{L}\left(T_{1}-T_{0}\right), Q_{2,0}=\beta_{1} M_{1} c_{L}\left(T_{2}-T_{1}\right), \cdots \\
& Q_{j, j-1}=\beta_{j-1} M_{j-1} c_{L}\left(T_{j}-T_{j-1}\right), Q_{j+1, j}=\beta_{j} M_{j} c_{L}\left(T_{j+1}-T_{j}\right), \cdots \\
& Q_{n-1, n-2}=\beta_{n-2} M_{n-2} c_{L}\left(T_{n-1}-T_{n-2}\right), Q_{L}=\dot{M} l\left(\frac{c_{p}\left(T_{C}-T_{S}\right)}{B_{T} l}-1\right)
\end{aligned}
$$

The exchange coefficients $\beta_{j}$ can be evaluated. The concept of exchange coefficient consists in simplifying the definition of the heat flux through the surface, by writing that it is proportional to the difference in temperature between the considered surface and another more or less distant surface. It is possible to evaluate the heat exchange coefficient by means of some assumptions. For the exchange between the kernel at $T_{0}$ and the first layer at $T_{1}$, we easily obtain $\beta_{0}=\kappa_{L} 3 r_{1} / r_{0}{ }^{2}\left(r_{1}-r_{0}\right)$. For the other coefficients, it is possible to compare the approximation of an exchange coefficient with the continuous purely conductive profile in the layer between $r_{j}$ and $r_{j+1}$. The thermal profiles are, for $r_{j}<r<r_{j+1}$ :

$$
T=\left\lfloor T_{j+1} r_{j+1}-T_{j} r_{j}-r_{j+1} r_{j}\left(T_{j+1}-T_{j}\right) / r\right\rfloor /\left.\left(r_{j+1}-r_{j}\right) \rightarrow(d T / d r)\right|_{r=r_{j}} ^{+}=r_{j+1}\left(T_{j+1}-T_{j}\right) / r_{j}\left(r_{j+1}-r_{j}\right) .
$$

Thus $Q_{j+1, j}=4 \pi k_{L} r_{j+1} r_{j}\left(T_{j+1}-T_{j}\right) /\left(r_{j+1}-r_{j}\right)$. Let us calculate $\beta_{j}$. We have $Q_{j+1, j}=\beta_{j} M_{j} c_{L}\left(T_{j+1}-T_{j}\right)=(4 / 3) \pi\left(r_{j}^{3}-r_{j-1}{ }^{3}\right) \rho_{L} c_{L} \beta_{j}\left(T_{j+1}-T_{j}\right)$. By comparison between these two expressions, one deduces $\beta_{j}=\kappa_{L} 3 r_{j+1} r_{j} /\left(r_{j}^{3}-r_{j-1}^{3}\right)\left(r_{j+1}-r_{j}\right)$. Setting $j=0, r_{j-1}=0$, one finds again the expression for $\beta_{0}$.

2. Case of layers of equal volumes

For the case in which the $\mathrm{n}$ layers have the same volume, one can write:

$$
\begin{aligned}
& \frac{d T_{0}}{d t}=\frac{n Q_{1,0}}{M c_{L}}, \frac{d T_{1}}{d t}=\frac{n}{M c_{L}}\left(Q_{2,1}-Q_{1,0}\right), \ldots \\
& \frac{d T_{n-2}}{d t}=\frac{n}{M c_{L}}\left(Q_{n-1, n-2}-Q_{n-2, n-3}\right), \frac{d T_{S}}{d t}=\frac{n}{M c_{L}}\left(Q_{L}-Q_{n-1, n-2}\right)
\end{aligned}
$$

with $r_{j, n}=\left(\frac{j+1}{n}\right)^{\frac{1}{3}} r_{s}$ for $0 \leq j \leq n-1$.

We model the interface fluxes in this way:

$$
Q_{j+1, j}=\beta_{j, n} \frac{M}{n} c_{L}\left(T_{j+1}-T_{j}\right)
$$

and then sum up these fluxes, which leads to

$d T_{0} / d t=\beta_{0, n}\left(T_{1}-T_{0}\right), d T_{1} / d t=\beta_{1, n}\left(T_{2}-T_{1}\right)-\beta_{0, n}\left(T_{1}-T_{0}\right) \ldots$.

and

$d T_{n-2} / d t=\beta_{n-2, n}\left(T_{n-1}-T_{n-2}\right)-\beta_{n-3, n}\left(T_{n-2}-T_{n-3}\right)$ with $T_{n-1}=T_{S}, \sum_{j=o}^{n-1} d T_{j} / d t=n Q_{L} / M c_{L}$

The previously defined heat exchange coefficients lead to the dimensionless relation $\Lambda_{j, n}=\theta n^{2 / 3} /\left[(j+1)^{-1 / 3}-(j+2)^{-1 / 3}\right], \Lambda_{j, n}=3 \beta_{j, n} \bar{\tau}_{v}$, with $\theta=9 \kappa_{L} \bar{\tau}_{v} / \bar{r}_{S}^{2}=\bar{\tau}_{v} / \tilde{\tau}_{T}$ as defined in Eq. (9).

\section{Linearized equations of the n-layers model}


For small perturbations, we can write:

$$
\begin{aligned}
& d T_{0}{ }^{\prime} / d t=\beta_{0, n}\left(T_{1}^{\prime}-T_{0}{ }^{\prime}\right), d T_{1}^{\prime} / d t=\beta_{1, n}\left(T_{2}{ }^{\prime}-T_{1}{ }^{\prime}\right)-\beta_{0, n}\left(T_{1}{ }^{\prime}-T_{0}{ }^{\prime}\right), \ldots d T_{n-2}{ }^{\prime} / d t=\beta_{n-2, n}\left(T_{n-1}{ }^{\prime}-T_{n-2}{ }^{\prime}\right) \\
& -\beta_{n-3, n}\left(T_{n-2}{ }^{\prime}-T_{n-3}{ }^{\prime}\right) \text { with } T_{n-1}{ }^{\prime}=T_{S}{ }^{\prime}, \Delta Q_{L}=\left(M c_{L} / n\right) \bar{T}_{S} \sum_{j=o}^{n-1} d T_{j}{ }^{\prime} / d t
\end{aligned}
$$

The linear character of the equations induces the following form: $\sum_{j=0}^{n-1} T_{j}^{\prime}=\sigma_{n}\left(\Lambda_{0, n}, \ldots, \Lambda_{n-2, n}, u\right) T_{S}^{\prime}$.

We calculate $\sigma_{n}=1+\underline{\Sigma}_{n-1}^{T} \underline{X}_{n-1}=1+\underline{\Sigma}_{n-1}^{T} \underline{\underline{M}}_{n-1}^{-1} \underline{e}_{n-1}$ using the matrix

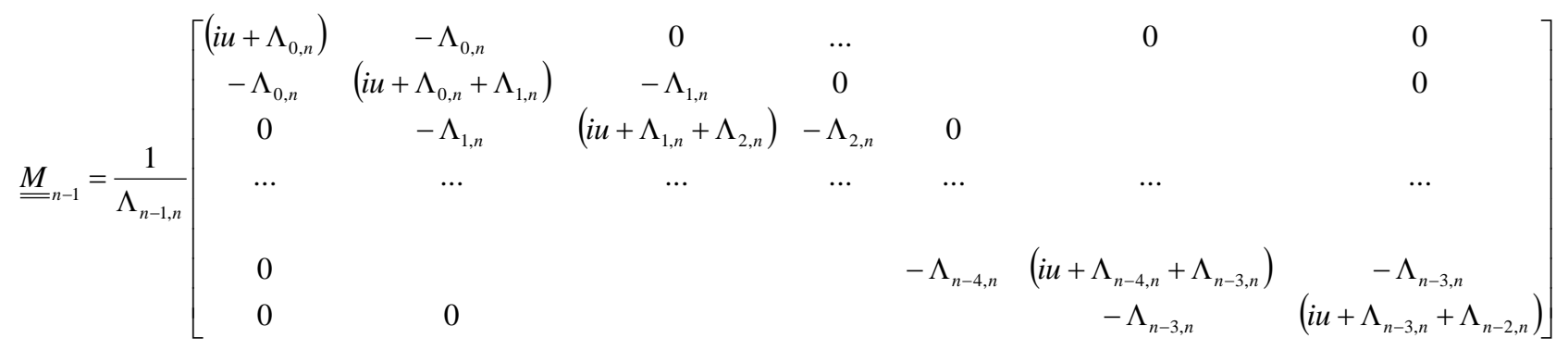

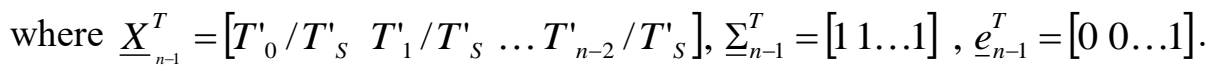

Setting $f^{\prime}=\hat{f}(r) e^{i \omega t}$ we find

$$
\begin{aligned}
& i \omega \hat{T}_{0}=\beta_{0, n}\left(\hat{T}_{1}-\hat{T}_{0}\right), i \omega \hat{T}_{1}=\beta_{1, n}\left(\hat{T}_{2}-\hat{T}_{1}\right)-\beta_{0, n}\left(\hat{T}_{1}-\hat{T}_{0}\right), \cdots \\
& i \omega \hat{T}_{j}=\beta_{j, n}\left(\hat{T}_{j+1}-\hat{T}_{j}\right)-\beta_{j-1, n}\left(\hat{T}_{j}-\hat{T}_{j-1}\right), \cdots, \Delta \hat{Q}_{L}=\frac{M c_{L}}{n} \bar{T}_{S} i \omega \sum_{j=0}^{n-1} \hat{T}_{j}
\end{aligned}
$$

\section{Transfer function of the Linearized $n$-Layer Model}

The transfer function $Z_{\mathrm{n}}$ has been obtained in the general case [25] and calculated up to $n=30$. From Appendix AII [Eqs. (A.7) and (A.8)] and from Eq. (13) one deduces

$$
Z_{n}=\frac{1}{\alpha} \frac{\hat{\dot{M}}}{\hat{p}_{C}}=\frac{i u}{1+i u} \frac{A-i u \frac{\sigma_{n}}{n}}{B+i u \frac{\sigma_{n}}{n}}
$$

where $\alpha$ is given by Eq. (A.5).

We verify that the cut-off frequency of the n-layer model with equal volumes converges towards that of the continuous model when the number of layers increases, as shown in Fig. 5. 


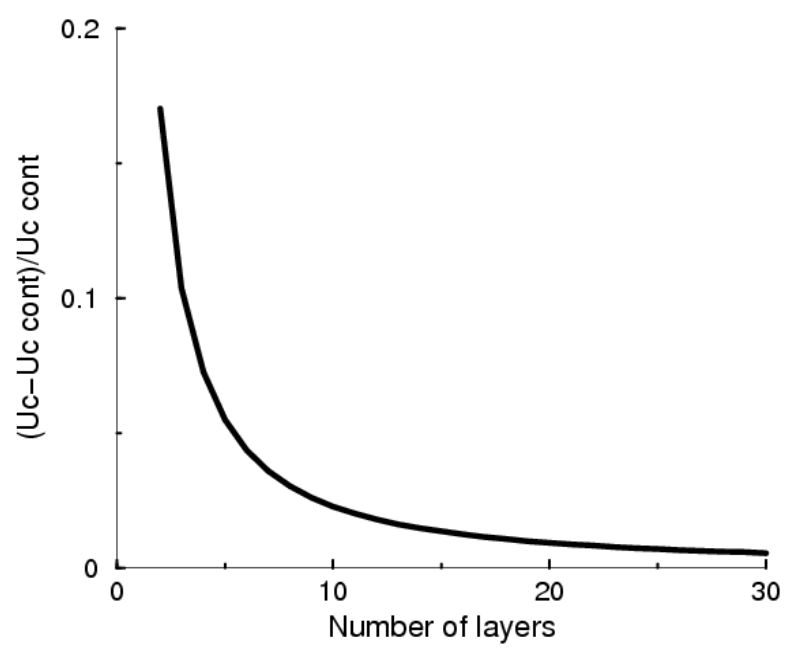

Fig. 5 Convergence of the n-layer model towards the continuous model for an increasing number of layers

(LOX droplet in a $\mathrm{O}_{2} / \mathrm{H}_{2} \mathrm{O}$ mixture, $T_{C}=550 \mathrm{~K}, p_{C}=10 \mathrm{~b}, Y_{A C}=0.9$ )

It can be seen that satisfactory results are obtained only with a relatively high number of layers (about 10 ), and that the convergence rate slows down with increasing $\mathrm{n}$. Nevertheless, it is possible to improve convergence by considering layers of different volumes and optimizing the relative volumes. Note that the conditions of this convergence test have been chosen so as to make the reduced exchange coefficient $\theta$ sufficiently high, which guarantees that the linearized continuous model can be used as a reference model.

\section{B. Two-Layer model}

The two-layer model is a particular case of the $n$-layer model with non equal volumes. We envisage this very simplified model for the purpose of low-cost calculations. The geometry of the two-layer model consists of two concentric spheres (Fig. 6). In the first one, of radius $r_{0}$, the temperature is supposed to be uniform and equal to $T_{0}$. Between the spheres of radii $r_{0}$ and $r_{I}=r_{S}$ the temperature is also uniform and equal to $T_{S}$. Temperatures $T_{0}$ and $T_{S}$ depend on time. Fig. 6 highlights the various heat exchanges. The equations of the problem are

$$
M_{0} c_{L} \frac{d T_{0}}{d t}=Q_{S 0}, \quad M_{S} c_{L} \frac{d T_{S}}{d t}=-Q_{S 0}+Q_{L}
$$

with

$$
\left\{\begin{array}{l}
Q_{S 0}=\beta_{0} M_{0} c_{L}\left(T_{S}-T_{0}\right), Q_{L}=\dot{M} \ell\left(\frac{C_{p}\left(T_{C}-T_{S}\right)}{l B_{T}}-1\right), M=\frac{4}{3} \pi r_{S}^{3} \rho_{L}, \\
M_{0}=\frac{4}{3} \pi r_{0}^{3} \rho_{L}, M_{1}=M_{S}=\frac{4}{3} \pi\left(r_{S}^{3}-r_{0}^{3}\right) \rho_{L}, \dot{M}=2 \pi \frac{k}{c_{p}} r_{S} N u^{*} \ln \left(1+B_{T}\right)
\end{array}\right.
$$

where $\rho_{L}$ is the liquid density. The heat exchange coefficient is

$$
\beta_{0}=\frac{3 \kappa_{L} r_{S}}{r_{0}^{2}\left(r_{S}-r_{0}\right)}=\frac{3 \kappa_{L}}{r_{S}^{2} \varepsilon^{2 / 3}\left(1-\varepsilon^{1 / 3}\right)}, \varepsilon=\left(\frac{r_{0}}{r_{S}}\right)^{3}
$$

we thus obtain

$$
\frac{d T_{0}}{d t}=\beta_{0}\left(T_{S}-T_{0}\right), \frac{d T_{S}}{d t}=-\frac{\varepsilon}{1-\varepsilon} \beta_{0}\left(T_{S}-T_{0}\right)+\frac{\dot{M} \ell}{M_{S} c_{L}}\left(\frac{\ln \left(1+B_{T}\right)}{B_{T} \ell}-1\right)\left(T_{C}-T_{S}\right)
$$




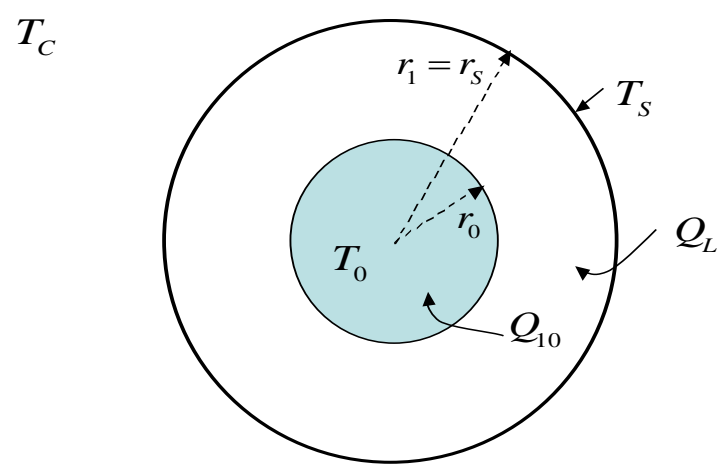

Fig. 6 Heat exchanges in the two-layer model

We evaluate the exchange coefficient $\beta_{0}$, as previously. Using Eq. (20) we can then obtain a relation between the approximate dimensionless exchange coefficient $\Lambda=3 \beta_{0} \bar{\tau}_{v}$ and the volume ratio:

$$
\Lambda \varepsilon^{2 / 3}\left(1-\varepsilon^{1 / 3}\right)=\theta
$$

used in the linearized theory below.

In steady mode of pure conduction and for constant radii $r_{0}$ and $r_{S}$, the temperature can be written $T=\left[T_{S} r_{S}-T_{0} r_{0}+\left(T_{0}-T_{S}\right) r_{S} r_{0} / r\right] /\left.\left(r_{S}-r_{0}\right) \rightarrow(d T / d r)\right|_{r=r_{S}}=r_{S}\left(T_{S}-T_{0}\right) / r_{0}\left(r_{S}-r_{0}\right)$.

With $Q_{10}=4 \pi h r_{0}^{2}\left(T_{S}-T_{0}\right)$, one deduces $h=r_{S} k_{L} / r_{0}\left(r_{S}-r_{0}\right), Q_{10}=4 \pi k_{L}\left(T_{S}-T_{0}\right) r_{S} r_{0} /\left(r_{S}-r_{0}\right)$.

With $Q_{10}=\beta_{0} M_{0} c_{L}\left(T_{S}-T_{0}\right)=(4 / 3) \pi r_{0}^{3} \rho_{L} c_{L} \beta_{0}\left(T_{S}-T_{0}\right)$, one deduces $\beta_{0}=\kappa_{L} 3 \bar{r}_{S} / \bar{r}_{0}^{2}\left(r_{S}-r_{0}\right)$. Since, in addition, $\theta=9 \kappa_{L} \bar{\tau}_{v} / \bar{r}_{S}^{2}=\bar{\tau}_{v} / \tilde{\tau}_{T}$ and $\Lambda=3 \beta_{0} \bar{\tau}_{v}=\theta \bar{r}_{S}^{3} / \bar{r}_{0}^{2}\left(\bar{r}_{S}-\bar{r}_{0}\right)$, with $\varepsilon=\left(\bar{r}_{0} / \bar{r}_{S}\right)^{3}$, we finally obtain equation (22).

\section{Linearized equations of the 2-layer model}

For small temperature perturbations, the above equations can be linearized and lead to:

$$
\frac{d T_{0}^{\prime}}{d t}=\beta_{0}\left(T^{\prime}{ }_{S}-T_{0}^{\prime}\right), \quad \bar{M} c_{L} \bar{T}_{S}\left[\varepsilon \frac{d T_{0}^{\prime}}{d t}+(1-\varepsilon) \frac{d T_{S}^{\prime}}{d t}\right]=\Delta Q_{L}
$$

where $\beta_{0}$ can be expressed as a function of $\varepsilon$ and $\theta$. Setting: $f^{\prime}=\hat{f}(r) e^{i \omega t}$, we find

$i \omega \hat{T}_{0}=\beta_{0}\left(\hat{T}_{S}-\hat{T}_{0}\right), \quad \Delta \hat{Q}_{L}=\bar{M} c_{L} \bar{T}_{S} i \omega\left(\varepsilon \hat{T}_{0}+(1-\varepsilon) \hat{T}_{S}\right)$

2. Transfer function of the two-layer model

The transfer function for the two-layer model is given by

$$
Z_{2}=\frac{1}{\alpha} \frac{\hat{\dot{M}}}{\hat{p}_{C}}=\frac{i u}{1+i u} \frac{(\Lambda+i u)(A-i u)-\varepsilon u^{2}}{(\Lambda+i u)(B+i u)+\varepsilon u^{2}}
$$

where $A, B, \varepsilon, \Lambda$ are the constant coefficients defined previously. The index of $Z$ indicates the number of layers in the discrete model for thermal exchanges inside the liquid droplet. 
As a first step, we will consider $A, B, \varepsilon, \Lambda$ as independent parameters [i.e., without Eq. (22)] for the case of a liquid Nusselt number equal to 2 . The liquid Nusselt number $N u$ characterizes the degree of agitation inside the liquid droplet, which may be induced by a small velocity difference between droplet and gas, or

by a non-homogeneous temperature at the droplet surface which can generate a Marangoni effect. For

$$
\varepsilon=0 \text { (one layer, } r_{0}=0 \text { ), we obtain again } Z_{2}=Z_{0} \text {. The cut-off frequency is thus } u_{c 0} \text {. }
$$

For $\varepsilon \neq 0$ and $\varepsilon \neq 1, \Lambda=0$, in this case there are two layers without any heat exchange, therefore:

$$
Z_{2}=\frac{i u}{1+i u} \frac{A-i u(1-\varepsilon)}{B+i u(1-\varepsilon)} \neq Z_{1}, \quad \frac{N_{2}}{\alpha}=\frac{u^{2}\left[A B+A+B-\varepsilon(A+B)-u^{2}(1-\varepsilon)^{2}\right]}{\left(1+u^{2}\right)\left[B^{2}+u^{2}(1-\varepsilon)^{2}\right]}
$$

and the reduced cut-off frequency becomes: $u_{c 2}^{2}=[A B+A+B-\varepsilon(A+B)] /(1-\varepsilon)^{2} \geq u_{c 1}^{2}$.

Thus, for a given droplet radius, the absence of heat exchange between the two layers leads to an extension of the amplification domain. The limiting case $\varepsilon=1$ corresponds to one layer without heat exchange. In this case, there is no cut-off frequency. In the general case of the two-layer model, we have $0<\varepsilon<1$.

Taking now into account the relation (22) between $\Lambda$ and $\varepsilon$, we obtain the results given in Fig. 7 for several values of $\theta$. From a qualitative point of view, the results are similar to those of Fig. 3 obtained with the continuous model. However, the stabilizing effect of thermal dissipation inside the droplet is under-estimated in comparison to the continuous model.

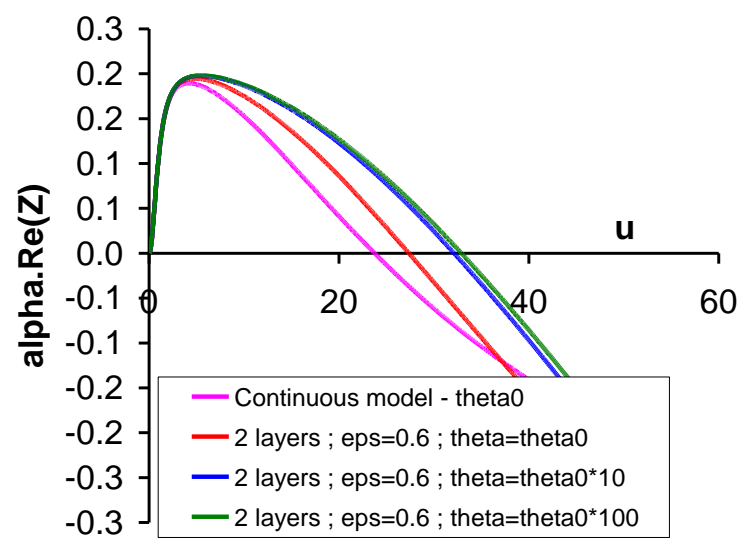

Fig. 7 Influence of the reduced exchange coefficient $\theta$ on the response factor of the 2-layer model $(\varepsilon=0.6)$, for the same system as Fig. 3 (LOX droplet in a $\mathrm{O}_{2} / \mathrm{H}_{2} \mathrm{O}$ mixture, $T_{C}=550 \mathrm{~K}, p_{C}=10 \mathrm{bar}, Y_{A C}=0.9, \theta_{0}=10.23$ ).

To obtain results in good agreement with those of the continuous model, we have performed an optimization of the parameter $\varepsilon$. We can notice that we are in the same mathematical situation as the nlayer model with layers of different volumes.

\section{Optimization of the Volume Ratio in the Two-Layer Model}

The goal of the optimization of the 2-layer model is to obtain, for any set of conditions, a behavior comparable to the one of the continuous model by choosing the size of the accumulation nucleus. The optimization parameter is the volume ratio.

We need to define a criterion to identify the best agreement with the continuous model. As a first criterion, it is important to find the best approximation for the cut-off frequency of the real part of the 
transfer function, so as to correctly predict the frequency range of amplifying or damping effect of unsteady vaporization. A second criterion is the response in the complex plane, including both amplitude and phase response. A comparison of the responses is presented in Fig. 8, which shows that in our validation case, evaluated for a LOX droplet, the best approximation with the 2-layer model is obtained for a volume ratio between 0.5 and 0.7 , whereas a model with 15 layers leads to a response much closer to the continuous model. We estimate the distance in the complex plane between the response of the 2-layer model and of the continuous model, in the range of interest $\left[0 ; u_{\text {sup }}\right.$, by a normalized mean quadratic error

Err $/ R_{\max }=\frac{1}{R_{\max }} \sqrt{\frac{\sum_{j=0}^{j=99}\left(Z_{2}\left(u_{\text {sup }} /(j+1)\right)-Z\left(u_{\text {sup }} /(j+1)\right)\right)^{2}}{100}}$, where $R_{\max }$ is the maximum value of the real part

of the transfer function of the continuous model. By means of this distance, we can check if the discrete model properly restitutes the global effect in this frequency range. We used a hybrid algorithm combining both criteria to manage eventual multiple solutions by the first criterion. Figure. 9 shows an example of result (optimized volume ratio and precision) obtained by the optimized 2-layer model, respectively with the distance criterion and the hybrid algorithm, in comparison to the n-layer model. This comparison is done by observing the sensitivity to the internal conductivity. The reference case for this sensitivity analysis is the one of a LOX droplet in an atmosphere at 10 bar and $550 \mathrm{~K}$. It can be seen that, in this case, the distance criterion and the hybrid criterion give very similar results, with acceptable error levels on the cut-off frequency but higher error levels on the normalized distance. However, the intrinsic drawback of the 2-layer model is that the optimization has to be performed for each set of chamber and propellants conditions. Concerning the n-layer model, tested here with 15 layers of equal volumes, we observe that its response is an order of magnitude closer to the continuous model, compared to the optimized 2-layer model. Moreover, this model does not need a systematic optimization, which is an important advantage.

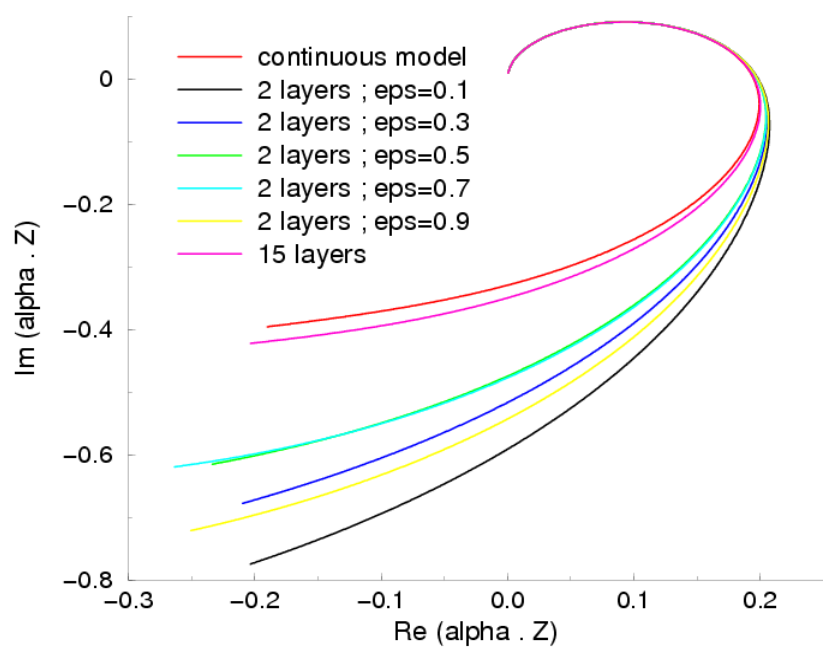

Fig. 8 Comparison in the complex plane of the responses of the different models (LOX droplet in a $\mathrm{O}_{2} / \mathrm{H}_{2} \mathrm{O}$ quiescent atmosphere, $T_{C}=550 \mathrm{~K}, p_{C}=10 \mathrm{bar}, Y_{A C}=0.9$, reduced frequency range $\left.\left[0 ; 2 u_{c}\right]\right)$. 

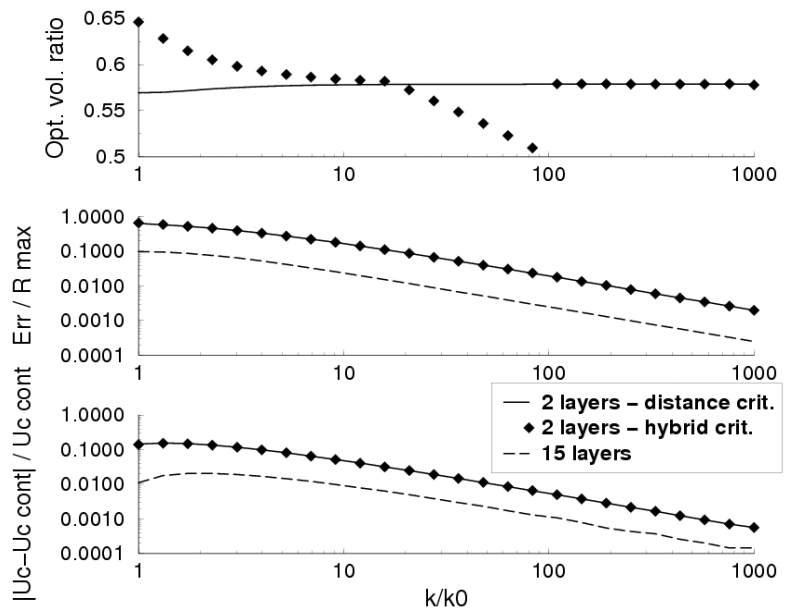

Fig. 9 Comparison of the precision of the optimized 2-layer model and the n-layer model - Influence of the internal conductivity (Reference case $k_{k} k_{0}$ : LOX droplet in a $\mathrm{O}_{2} / \mathrm{H}_{2} \mathrm{O}$ mixture, $T_{C}=550 \mathrm{~K}, p_{C}=10 \mathrm{~b}, Y_{A C}=0.9$ )

\section{Conclusions}

An investigation of the unsteady evaporation of a propellant droplet submitted to an acoustic excitation, undertaken in the framework of liquid propellant rocket engines high frequency stability analysis, shows the importance of internal thermal exchanges. A continuous analytical model has been established in the frame of the Heidmann analogy, which represents a "mean droplet" at a fixed chamber location in the established regime. We have then developed a discrete $n$-layer model for implementation in a CFD code. This discrete model has been validated by comparison with the continuous model which can be considered as a reference in the limit of small perturbations and when the characteristic time of thermal conduction is much smaller than the droplet lifetime. We assume that the results of this validation remain valid outside the linear domain.

The model with $\mathrm{n}$ layers of equal volumes is sufficiently precise for $n \geq 10$, but the CPU cost will be relatively high. In order to obtain a better trade-off between precision and cost, we have considered a nlayer model with a reduced number of layers in which the volume of each layer has to be optimized. This method has been tested with the 2-layer model.

This model of a droplet with two layers of optimized volume ratio reproduces the droplet response to a pressure perturbation in a reasonably realistic way, with a low CPU cost. However, the volume optimization has to be carried out for each situation, taking into account the flow conditions, the thermal properties of the propellants, chamber thermodynamics and chemistry, and atomization.

In the future, we intend to implement the multi-layer model (in a nonlinear form) into a CFD code and work on other aspects of the modeling in order to validate and optimize tools for an enlarged range of conditions. Specific aspects include 1) the influence of external convection, 2) the case where the characteristic time for thermal conduction is comparable to the droplet lifetime, and 3) the case of a velocity perturbation (an acoustic pressure node). 


\section{Appendix - Linearized Equations of the Gas Phase in the Context of Quasisteady Assumptions}

\section{Gas Phase Equations for Evaporation}

Let us consider the gas phase around a vaporizing droplet. The classical quasi-steady-state hypothesis can be extended to droplet vaporization in the presence of an external flow, with exchanges of mass, momentum and energy between the droplet and the external flow, as was done by Abramzon and Sirignano in [26]. The gas phase equations are modified to account for these exchanges. We suppose an ideal mixture of perfect gases.

This is nearly the classical problem ([27-29]) but with heat exchange between the gas and the liquid droplet. In the gas field, the solution of the diffusion equation is the same as the one without heat-up of the droplet, and gives for the mass flow rate of the droplet:

$$
\dot{M}=2 \pi \rho D r_{S} S h^{*} \ln \left(1+B_{M}\right) \text {, with } B_{M}=\left(Y_{F S}-Y_{F C}\right) /\left(1-Y_{F S}\right)
$$

where $\rho$ is the gas density, $D$ the mass diffusion coefficient, $S h *$ the Sherwood number introduced by Abramzon and Sirignano [26] in an extended film model, $Y_{j}$ the mass fraction of species $j$. The subscripts $F, S$, and $C$, represent respectively the fuel, the droplet surface and the conditions far from the droplet.

On the other hand, solution of the conduction equation is modified because of the change of the boundary condition at the droplet surface. We introduce the heat flux $Q_{L}$ which characterizes the heat given to the drop, in addition to that necessary for evaporation, and a Nusselt number $N u$ (Note that, in the following, the Nusselt and Sherwood numbers will be both taken equal to 2). The mass flow rate is then given by

$$
\dot{M}=2 \pi \frac{k}{c_{p}} r_{S} N u * \ln \left(1+B_{T}\right), \text { with } B_{T}=c_{p}\left(T_{C}-T_{S}\right) /\left(\ell+Q_{L} / \dot{M}\right)
$$

from which we can deduce $Q_{L}=\dot{M} \ell\left(B_{T 0} / B_{T}-1\right), B_{T 0}=c_{p}\left(T_{C}-T_{S}\right) / \ell$. The Spalding parameters $B_{T}$ and $B_{T 0}$ (noted so because is corresponds to the case $Q_{L}=0$ ) depend on the droplet temperature $T_{S}$, on the temperature at infinity $T_{C}$, on the gas specific heat at constant pressure $c_{p}$, and on the latent heat per unit mass $\ell . k$ is the heat conductivity of the gas, which can be replaced by an effective thermal conductivity as in [26]. The mass flow rate is related to the droplet mass $M$ by

$$
\dot{M}=-d M / d t, M=\frac{4}{3} \pi r_{S}^{3} \rho_{L}
$$

where $t$ is the time and $r_{S}$ the droplet radius.

The Spalding parameter for heat exchange $B_{T}$, defined by (A.2), is connected to the Spalding parameter for mass exchange $B_{M}$, defined by (A.1), by the following equation:

$$
\dot{M}=2 \pi \frac{k}{c_{p}} r_{S} N u * \ln \left(1+B_{T}\right)=4 \pi \rho D r_{S} S h * \ln \left(1+B_{M}\right)
$$

which is derived from (A.1) and (A.2). So $B_{T}$ is a function of $B_{M}$, which depends on the gaseous fuel mass fraction at the droplet surface (for a droplet and its surrounding atmosphere at rest, and if the Lewis number Le is equal to unity, we have $B_{T}=B_{M}$ ). This mass fraction is connected to temperature $T_{S}$ by the equilibrium relation between molar free energies of the liquid and the gas respectively: $\mu_{L}=\mu_{F}$. When $\mu_{L}$ is function of $T$ only, and for an ideal gas mixture, this leads to $p X_{F S}=p_{s a t}\left(T_{S}\right)$, where $\mathrm{p}$ is the pressure, $X_{j}$ is the molar fraction of species $\mathrm{j}$ in the mixture and where the saturated vapor pressure can be written [22] : $p_{\text {sat }}\left(T_{S}\right)=\exp \left(a-b /\left(T_{S}-c\right)\right)$, with $a, b$ and $c$ constant coefficients.

Then, we can relate $Y_{F S}$ to the surface molar fraction $X_{F S}$, thus the surface mass fraction $Y_{F S}$ appears as a function of surface temperature $T_{S}$ and total pressure $p$, which is assumed uniform and constant: $Y_{F S}=f\left(T_{S}, p\right)$. Thus $B_{T}$ and $B_{M}$ are function of $T_{S}$ only. 
The latent heat itself is function of temperature and concentration at the surface. Indeed, from the Clapeyron relation, one has per mole of pure substance: $L=\left(R T^{2} / p\right)(d p / d T)_{\text {sat }}$. This relation, applied here to the unit mass, and replacing the pressure by the partial pressure, gives

$$
\ell=\frac{b R T_{S}^{2}}{M_{F}\left(T_{S}-c\right)^{2}}
$$

where $M_{j}$ is the molar mass of species $j$ and $A$ a diluted species (the gaseous mixture is made of $A$ and $F$, the droplet is made of pure liquid $F$ ). As temperature and concentrations are not constant in the environment of the droplet, the averaged properties $c_{p}, k$ are evaluated at a reference temperature and composition $\bar{T}=T_{S}+A_{r}\left(T_{C}-T_{S}\right), \bar{Y}_{F}=Y_{F S}+A_{r}\left(Y_{F C}-Y_{F S}\right)$, where $A_{r}=1 / 3$ is generally chosen.

\section{Small Perturbation Equations of the Gas Phase}

Consider now small acoustic perturbations, writing $f=\bar{f}+\Delta f$ where $f$ is a flow parameter, $\Delta f$ is the absolute perturbation, and $f^{\prime}=\Delta f / f$ is the corresponding relative perturbation.

We assume Heidmann's configuration, i.e. a mean droplet fed by liquid $F$ with a mass flow rate $\overline{\dot{M}}$ in the stabilized regime (Fig.1). The Eq. (A.3) is then replaced by Eq. (1). The velocity perturbation is assumed equal to zero and we look for the relation between the imposed chamber perturbation $p$ ' and the resulting mass flow rate perturbation $\dot{M}^{\prime}$. To do this, we have to write the equations of both the gas phase and the liquid. Here we derive the equations for the gas phase. The equations for the liquid phase where given in Sec. III.

From: $\dot{M}=4 \pi \rho D r_{S} \ln \left(1+B_{M}\right)$, with

we obtain

$$
B_{M}=\frac{Y_{F S}-Y_{F C}}{1-Y_{F S}}
$$

$$
\dot{M}^{\prime}=\frac{1}{3} M^{\prime}+\frac{\bar{B}_{M}}{\left(1+\bar{B}_{M}\right) \ln \left(1+\bar{B}_{M}\right)} B_{M}{ }^{\prime}
$$

with

$$
B_{M}{ }^{\prime}=\frac{\bar{Y}_{A C} \bar{Y}_{F S}}{\bar{Y}_{A S}\left(\bar{Y}_{F S}-\bar{Y}_{F C}\right)} Y_{F S}{ }^{\prime}
$$

And

$$
Y_{F S}^{\prime}=\frac{\mathcal{M}_{F}}{\mathcal{M}_{F} \bar{X}_{F S}+\mathcal{M}_{A} \bar{X}_{A S}} X_{F S}^{\prime}
$$

$$
X_{F S}{ }^{\prime}=p_{s a t}{ }^{\prime}-p_{C}{ }^{\prime}, p_{s a t}{ }^{\prime}=\bar{b} T_{S}{ }^{\prime}
$$

$$
\bar{b}=\frac{b \bar{T}_{S}}{\left(\bar{T}_{S}-c\right)^{2}}
$$

The quantity $Q_{L}$, the heat flux for heating-up the droplet, is zero for the stabilized reference state. This precludes the use of a relative perturbation $Q_{L}{ }^{\prime}$, and it is necessary to keep the absolute perturbation $\Delta Q_{L}$. From: $Q_{L}=\dot{M} l\left[c_{p}\left(T_{C}-T_{S}\right) / B_{T} l-1\right], l=b R T_{S}^{2} / M_{F}\left(T_{S}-c\right)^{2}$, one deduces

$\Delta Q_{L}=-\overline{\dot{M}} \bar{\ell}\left(\frac{\bar{T}_{S}}{\bar{T}_{C}-\bar{T}_{S}} T^{\prime}{ }_{S}-\frac{\bar{T}_{C}}{\bar{T}_{C}-\bar{T}_{S}} T_{C}^{\prime}+B_{T}^{\prime}+\ell^{\prime}\right)$

With

$$
B_{T}{ }^{\prime}=\frac{\overline{B_{M}}}{\overline{L e}} \frac{\left(1+\overline{B_{M}}\right)^{\frac{1}{\overline{L e}}-1}}{\left(1+\overline{B_{M}}\right)^{\frac{1}{\bar{L}}}-1} B_{M}{ }^{\prime}, l^{\prime}=-\frac{2 c}{\overline{T_{S}}-c} T_{S}{ }^{\prime}
$$


Eliminating the intermediate parameters, one deduces

$$
\frac{d \dot{M}^{\prime}}{d t}+\frac{\dot{M}^{\prime}}{3 \bar{\tau}_{v}}=\alpha\left(\bar{b} \frac{d T_{S}^{\prime}}{d t}-\frac{d p_{C}^{\prime}}{d t}\right)
$$

with

$$
\begin{gathered}
\alpha=\frac{\bar{B}_{M}}{\left(1+\bar{B}_{M}\right) \ln \left(1+\bar{B}_{M}\right)} \frac{\bar{Y}_{A C} \bar{Y}_{F S}}{\bar{Y}_{A S}\left(\bar{Y}_{F S}-\bar{Y}_{F C}\right)} \frac{\mathcal{M}_{F}}{\mathcal{M}_{F} \bar{X}_{F S}+\mathcal{M}_{A} \bar{X}_{A S}} \\
\bar{\tau}_{v}=\frac{\bar{M}}{\overline{\dot{M}}}=\frac{\rho_{L} c_{p} \bar{r}_{S}^{2}}{3 k \ln \left(1+\bar{B}_{T}\right)}
\end{gathered}
$$

and

$$
\Delta Q_{L}=\overline{\dot{M}} \bar{\ell}\left(\bar{a} p_{c}{ }^{\prime}-\mu T_{S}{ }^{\prime}\right)
$$

with

$$
\begin{gathered}
\lambda=\frac{c_{L} \bar{T}_{S}}{\bar{\ell}} \\
\mu=\frac{\bar{T}_{S}}{\bar{T}_{C}-\bar{T}_{S}}-\frac{2 c}{\bar{T}_{S}-c}+\bar{b} \varphi \\
\bar{b}=\frac{\bar{T}_{S}}{\left(\bar{T}_{S}-c\right)^{2}} b \\
\bar{a}=\frac{\bar{T}_{C}}{\bar{T}_{C}-\bar{T}_{S}} \frac{\gamma-1}{\gamma}+\varphi
\end{gathered}
$$

And

$$
\varphi=\frac{\bar{B}_{M}}{L e} \frac{\left(1+\bar{B}_{M}\right)^{\frac{1}{L e}-1}}{\left(1+\bar{B}_{M}\right)^{\frac{1}{L e}}-1} \frac{\bar{Y}_{A C} \bar{Y}_{F S}}{\bar{Y}_{A S}\left(\bar{Y}_{F S}-\bar{Y}_{F C}\right)} \frac{\mathcal{M}_{F}}{\mathcal{M}_{F} \bar{X}_{F S}+\mathcal{M}_{A} \bar{X}_{A S}} .
$$

For a Lewis number equal to 1 we have

$$
\varphi=\frac{\bar{Y}_{A C} \bar{Y}_{F S}}{\bar{Y}_{A S}\left(\bar{Y}_{F S}-\bar{Y}_{F C}\right)} \frac{\mathcal{M}_{F}}{\mathcal{M}_{F} \bar{X}_{F S}+\mathcal{M}_{A} \bar{X}_{A S}} \cdot
$$

For the case of harmonic perturbations one has: $f^{\prime}=\hat{f} e^{i \omega t}$ for any relative small perturbation and: $\Delta Q_{L}=\Delta \hat{Q}_{L} e^{i \omega t}$ for the absolute heat flux perturbation. Consequently, the two equations of the gas phase become:

$$
\hat{\dot{M}}=\alpha \frac{i u}{1+i u}\left(\bar{b} \hat{T}_{S}-\hat{p}_{C}\right)
$$

with $u=3 \bar{\tau}_{v} \omega$

and

$$
\Delta \hat{Q}_{L}=\overline{\dot{M}} \bar{\ell}\left(\bar{a} \hat{p}_{C}-\mu \hat{T}_{S}\right)
$$

\section{Acknowledgments}

This work was performed as part of the French-German research program on rocket engine instabilities. It was funded by the Centre National d'Etudes Spatiales (CNES) and the Office National d'Etudes et Recherches Aerospatiales (ONERA) in the framework of their Research and Technology program. 
The authors are grateful to Dr. Geoff Searby, Research Director at CNRS and Member of the Scientific Committee of the abovementioned French-German program, for his kind scientific remarks.

\section{References}

1. Sirignano, W. A., Delplanque, J.-P., Chiang, C.H. \& Bhatia, R., "Liquid-propellant droplet vaporization: a rate controlling process for combustion instability", in Liquid rocket engine combustion instability, edited by V. Yang, W. E. Anderson, Progress in Astronautics and Aeronautics Vol. 169, AIAA, Washington, D.C., 1994.

2. De Benedictis M., «Instabilités couplées haute fréquence dans les moteurs-fusées à ergols liquides : étude du couplage chambre de combustion / système d'alimentation », $\mathrm{PhD}$. Thesis presented at the Univ. of Poitiers, France, Dec. 2007.

3. Bhatia, R. \& Sirignano, W. A., "One-dimensional analysis of liquid-fueled combustion instability", Journal of Propulsion and Power Vol. 7, N 6, 1991, pp. 953-961. doi:10.2514/3.23413

4. Delplanque, J.-P. \& Sirignano, W. A. (1994): "Boundary-layer stripping effects on droplet transcritical convective vaporization", Atomization and sprays, 4, pp. 325-349.

5. Delplanque, J-P. \& Sirignano, W. A. (1996): "Transcritical liquid oxygen droplet vaporization: effect on rocket combustion instability", Atomization and sprays, 4, pp. 325-349.

6. DiCicco, M. and Buckmaster, J. (1994): "Acoustic instabilities driven by slip between a condensed phase and the gas phase in combustion systems", 32 ${ }^{\text {nd }}$ AIAA Aerospace Sciences Meeting and Exhibit, Paper AIAA 940103, Reno, NV (USA), January pp. 10-13.

7. Dubois, I., Habiballah, M. and Lecourt, R. (1995): "Numerical analysis of liquid rocket engine combustion instability", 33 ${ }^{\text {rd }}$ AIAA Aerospace Sciences Meeting and Exhibit, Paper AIAA-95-0607, Reno, NV (USA) January pp. 9-12.

8. Duvur, A., Chiang, C.H. \& Sirignano, W. A. (1996): "Oscillatory fuel droplet vaporization: driving mechanism for combustion instability", Journal of Propulsion and Power, 12, n², pp. 358-365. doi:10.2514/3.24036

9. Fachini, F.F. (1998): "Transient effects in the droplet combustion process in an acoustically perturbed high temperature environment", Combust. Sci. and Tech., 139, pp. 173-189. doi:10.1080/00102209808952086

10. Heidmann, M. F. \& Wieber P. R., "Analysis of frequency response characteristics of propellant vaporization", NASA Technical Note D-3749, 1966.

11. Heidmann, M. F., "Frequency response of a vaporization process to distorted acoustic disturbances", NASA Technical Note D-6806, 1972.

12. Laroche, E., Habiballah, M. \& Kuentzmann, P., "Numerical analysis of liquid rocket combustion instability; preliminary 3D acoustic calculations", $36^{\text {th }}$ AIAA/ASME/SAE/ASEE Joint Propulsion Conference and Exhibit, 16-19 July, Huntsville, Alabama, AIAA 2000-3497, 2000.

13. Tong, A. Y. \& Sirignano, W. A., "Oscillatory vaporization of fuel droplets in an instable combustor", J. Propulsion, 5, n³, 1989, pp. 257-261. doi:10.2514/3.23146

14. Wieber, P.R. \& Mickelsen, W.R., "Effect of transverse acoustic oscillations on the vaporization of a liquid-fuel droplet", NASA Technical Note D-287, 1960.

15. Williams, F. A., "Combustion processes and instabilities in liquid-propellant rocket engines", Rept. A190024, 29, Ja n. 2004.

16. Rayleigh, Lord, "The theory of sound", Macmillan, London, 1945

17. Harrje D.T., and Reardon F.H., Liquid propellant rocket combustion instability, NASA SP-194, 1972

18. Tong, A. Y. \& Sirignano, W. A., "Oscillatory vaporization of fuel droplets in an instable combustor", J. Propulsion, 5, $\mathrm{n}^{\circ} 3,1989$, pp. 257-261.

19. Crespo, A. \& Liñan, A., "Unsteady effects in droplet evaporation and combustion", Combustion Science and Technology, 11, 1975, pp. 9-18. doi:10.1080/00102207508946679

20. Lefebvre, A.H., “Atomization and sprays”, Hemisphere Publishing Corporation, New York, 1989.

21. Strahle, W.C., "A theoretical study of unsteady droplet burning: transient and periodic solutions", Aeronautical Engineering Report 671, Princeton University (NASA CR-55516), 1963.

22. Chin, J. S. \& Lefebvre A. H., "The role of the heat-up period in fuel drop evaporation", Int. J. Turbo Jet Engines, 2, 1985, pp. 315-325. 
23. Law, C. K. \& Sirignano, W. A., "Unsteady droplet combustion with droplet heating-II: conduction limit", Combustion and flame, 28, 1977, pp. 175-186. doi:10.1016/0010-2180(77)90023-2

24. Prud'homme, R., Flows of reactive fluids Springer FMIA Series Springer-Birkhaüser, Boston 2010 (to be published).

25. Matuszewski, L., "Evaporation instationnaire de gouttes d'ergols", Rapport de stage ONERA/Ecole Polytechnique, 2006.

26. Abramzon, B. \& Sirignano, W. A., "Droplet vaporization model for spray combustion calculations", Int. J. Heat Mass Transfer, 32, No 9, 1989, pp. 1605-1618. doi:10.1016/0017-9310(89)90043-4

27. Godsave, G.A.E., "Studies of the combustion of drops in a fuel spray. The burning of single drops of fuel", Fourth Symposium (International) on Combustion, The Combustion Institute, 1953, pp. 818-830.

28. Spalding, D. B., "Studies of the combustion of drops in a fuel spray. The burning of single drops of fuel", Fourth Symposium (International) on Combustion, The Combustion Institute, 1953, pp. 847-864.

29. Williams, F.A. (1985): Combustion theory, $2^{\text {nd }}$ ed., The Benjamin Cumming Publishing, Menlo Park, CA

Published in:

JOURNAL OF PROPULSION AND POWER

Vol. 26, $\mathrm{N}^{\circ}$. 1, January-February 2010

p. $74-83$ 